Voix et Images

volxetimages

\title{
Charles Guérin : Des fonctions ou une fonction...
}

\section{Janine Frot}

Volume 4, numéro 2, décembre 1978

Guy Lafond

URI : https://id.erudit.org/iderudit/200156ar

DOI : https://doi.org/10.7202/200156ar

Aller au sommaire du numéro

Éditeur(s)

Les Presses de l'Université du Québec

ISSN

0318-9201 (imprimé)

1705-933X (numérique)

Découvrir la revue

Citer cet article

Frot, J. (1978). Charles Guérin : Des fonctions ou une fonction... Voix et Images, 4(2), 258-263. https://doi.org/10.7202/200156ar d'utilisation que vous pouvez consulter en ligne.

https://apropos.erudit.org/fr/usagers/politique-dutilisation/ 


\section{Charles Guérin :}

\section{Des fonctions ou une fonction...}

Le texte littéraire apparaît dorénavant comme un univers structuré, organisé sur la base des principes rigoureux et constitué d'un ensemble de règles logico-sémantiques stables et communes à tous les textes. Des théoriciens tels que Bremond, Todorov, Barthes, Souriau, Greimas ont élaboré, à la suite des travaux de Propp qui, en 1928, mettait en évidence une structure commune dans un échantillon de cent contes russes, des modèles de description valables pour tous les textes.

Ces recherches allaient, bien entendu, modifier les méthodes traditionnelles d'analyse des textes littéraires et donner lieu parfois à des excès tels que loin d'apparaître comme des instruments facilitant l'ouverture et la compréhension du texte, les modèles, parce que non assimilés et par suite mal utilisés, allaient faire figure de moyens d'incarcéràtion, le texte apparaissant sous leur emprise réduit, figé dans un cadre rigide et ceci dans un but non clairement défini.

C'est bien ainsi que nous apparaît le roman de Chauveau, Charles Guérin, dont le texte se présente dans l'édition de mars 1973 précédé d'une analyse ${ }^{1}$ fondée sur les travaux de Propp, analyse tendant à démontrer que ce roman est assimilable, par sa structure, à un conte russe. Nous allons, en utilisant cet exemple d'analyse, observer dans quelle mesure et à quel degré la méthode d'analyse a ou n'a pas été suivie et quelles en sont les conséquences.

Les travaux de Propp, rappelons-le, visent à mettre en évidence la présence de certains invariants dans le texte. Sa recherche porte sur une partie du texte seulement, soit la mince couche narrative qu'est le récit; par ailleurs, son champ de recherche, qui ne retient qu'un aspect de la littérature à savoir le conte, porte sur un échantillon de cent contes merveilleux russes. Les invariants mis à jour sont les actions des personnages dénommées fonctions et qui apparaissent, selon Propp, enchaînées les unes aux autres de façon unilinéaire et dans un ordre strict, de sorte que chaque conte est constitué d'une unique séquence de fonctions. Tous les contes merveilleux russes s'articulent ainsi autour d'un schéma de base qui est le squelette du conte. 
C'est donc ce modèle rigide constitué d'une chaîne de 31 fonctions qui est appliqué au roman de Chauveau. Dans un premier temps, certaines données linguistiques sont extraites du texte en vue de retrouver à partir du modèle de Propp une structure identique dans Charles Guérin. Les travaux de Propp sont assez connus pour que nous puissions nous dispenser d'énumérer les 31 fonctions qui constituent la séquence du conte; d'autre part, la caractéristique fondamentale de ces fonctions étant leur enchaînement logique et nécessaire, il nous suffira d'opérer une coupe dans le matériel d'analyse du texte de Chauveau pour obtenir un échantillon représentatif. Nous retiendrons donc les fonctions clés où sont manifestés affrontement des sujets et communication de l'objet de la quête, soit les fonctions numérotées de 15 à 19 et identifiées par Propp dans cet ordre:

15 - Le héros est conduit près de l'objet de sa quête.

16 - Affrontement du héros et de l'agresseur.

17 - Le héros reçoit une marque ou un gage.

18 - L'agresseur est vaincu.

19 - Méfait initial réparé.

L'analyse, pour chacun de ces postes, tire du texte de Chauveau les données linguistiques suivantes:

15 - "Charles se rend donc avec son don chez Marichette».

16 - Néant.

17 - Néant.

18 - L'agresseur est vaincu : fonction J6 (expulsion); en effet, l'auteur note: «Nous ne sommes point certain, malgré son habileté, qu'il [Voisin] fasse la conquête de cette dot, pour peu que l'occasion tarde à se présenter. Les années qui s'écoulent n'ajoutent point de charme à sa physionomie qui de laide est devenue affreuse ..."

19 - Méfait initial réparé «pour Charles qui va s'installer sur une terre nouvelle le méfait est réparé ".

Une lecture correcte de cette suite de fonctions nous permet d'affirmer que l'objet de la quête est Marichette, objet qui polarise le désir de deux sujets dont l'un, Charles Guérin, est le héros tandis que l'autre, Voisin, est l'antihéros ou l'agresseur. L'objet étant attribué au héros, l'antihéros se trouve dépossédé et le méfait initial est ainsi réparé.

Or, une lecture de Charles Guérin révèle que Voisin n'a aucun contact avec Marichette qu'il ne connaît point; le seul objet qui l'intéresse c'est Clorinde Wagnaër, la fille du riche Wagnaër dont il convoite les biens. II n'y a donc aucun lien entre la fonction 15 et la fonction 18 puisque les deux sujets en relation d'attribution avec des objets différents n'apparaissent pas dans un rapport d'opposition. II n'y a plus de héros et d'antihéros, il n'y a que deux héros ou deux sujets poursuivant leur quête dans des directions différentes. II n'est donc pas surprenant que la fonction 16 ayant trait à l'affrontement soit absente. 
Si Marichette n'est pas à la croisée des chemins de ces deux sujets, Clorinde par contre s'y trouve. Charles Guérin reçoit même de sa mère le mandat très explicite de faire sa conquête: "Elle est donc bien aimable cette Clorinde qu'elle t'a ensorcelé du premier coup? s'étonne-t-elle, ajoutant aussitôt : si tu savais comme cela me fait plaisir, [ce mariage] serait là, après tout, un bon moyen de finir toutes les difficultés ${ }^{2}$. 》

Maintenir Voisin sur la trajectoire de l'agresseur implique que Clorinde Wagnaër soit l'objet de la quête quand le héros est Charles Guérin. Les fonctions 16 et 17 sont alors susceptibles de recevoir un contenu puisque l'affrontement se termine par la dépossession totale de Charles Guérin qui recevra une "marque" puisque Clorinde désireuse de lui manifester sa fidélité entre au couvent, sûre du même coup d'échapper à Voisin. Si le héros reçoit un gage d'amour, il n'en demeure pas moins vaincu car dépossédé de tous ses biens. L'agresseur lui aussi se trouve vaincu non par suite de l'affrontement avec le héros qu'il a dépossédé mais par Dieu qui lui ravit l'objet de sa quête et le dépossède. II est donc illogique d'admettre que le méfait est réparé puisque le méfait initial se trouvait être un processus de dépossession, amorcé par Wagnaër, vis-à-vis de la famille Guérin, processus qui se complète ainsi que nous l'avons vu à la fonction 16. L'analyse est d'autant plus incohérente que cette dépossession de la famille Guérin y est reconnue dans la fonction "méfait initial ».

Dans l'analyse de Charles Guérin, la présence de fonctions erratiques, qui ont pour effet de rompre la logique de la séquence, est attribuable en partie à l'absence de découpage du texte, étape préliminaire et fondamentale de toute analyse. Les enseignements de Propp à cet égard sont explicites: “Un conte, dit-il, peut être composé de plusieurs mouvements et l'analyse d'un texte exige TOUT D'ABORD qu'on en détermine le nombre ${ }^{3}$."

Alors que plusieurs séquences élémentaires se développent et progressent de front situant en des plans distincts diverses intrigues dont celle qui se noue entre Charles et Marichette, celle qui se noue entre Charles et Clorinde ou encore la quête de Wagnaër ainsi que la conquête de la Terre, ensemble complexe dont la physionomie étagée pourra être ainsi représentée :

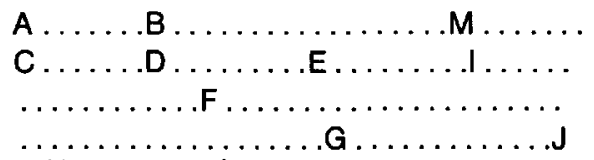

L'analyse, excluant certains événements et en sélectionnant d'autres arbitrairement en donne une représentation unilinéaire réduite et de type :<smiles>[2H][13B]CCCC[12CH]</smiles>

Ainsi les fonctions des différentes séquences ont été mélangées et la chaîne obtenue est une reconstitution d'un récit à partir d'une sélection arbitraire. 
Outre cette absence de découpage qui entraîne la fabrication d'un ensemble incohérent, la distinction entre niveaux discursif et narratif n'est pas faite, ce qu'atteste la fonction 18: «agresseur vaincu» et pour laquelle les données linguistiques équivalentes ne correspondent aucunement à l'action d'un personnage mais à des commentaires du narrateur, commentaires exprimés au temps présent et par conséquent situés hors du récit. La notion de fonction est pourtant clairement posée par Propp qui l'entend «comme l'action du personnage définie du point de vue de sa portée significative dans le déroulement du récit ${ }^{4}$ ". Cet exemple n'est d'ailleurs pas isolé, comme en témoigne la fonction 2 ainsi identifiée par Propp : "Le héros se fait signifier une interdiction ou un ordre» et pour laquelle l'analyse pose : "La mère Guérin propose aux deux frères de faire attention...m. Si nous nous reportons au texte, nous remarquons que Madame Guérin se borne à donner quelques conseils de ce genre: «[...] n'oubliez pas d'entrer chez tous les curés... pour vous réchauffer et vous reposer... Promettez-moi bien de ne pas continuer la route si vous êtes trempés...". Ce ne sont là "que menus actes qui forment le tissu du premier niveau" et non les grandes articulations de la praxis ${ }^{5}$. Ces conseils, riches d'indices concernant Madame Guérin, sont nuls d'un point de vue fonctionnel. Par contre, quand Madame Guérin émancipe Charles ou quand elle lui conseille d'épouser Clorinde ce sont des fonctions qui orientent la suite du récit mais qui ne paraissent nullement dans ce schéma.

Malgré ce double handicap, l'analyse est formelle quant à sa conclusion: Charles Guérin offre une parenté de structure avec le conte russe. Or, ce qui est caractéristique dans la chaîne de fonctions obtenue par Propp c'est un ordre: dans le conte merveilleux la «succession des fonctions est toujours identique ${ }^{6}$. Ainsi la Transfiguration du héros (fonction 29) précède toujours le mariage ou toute autre forme d'attribution d'une récompense, ce qui n'est pas le cas dans la présente analyse puisque Marichette, vue comme l'objet de la quête, est offerte au héros dans un premier temps tandis que ce n'est que bien plus tard qu'a lieu une Transfiguration, véritable démonstration de la valeur individuelle du héros. Cette deuxième phase est sans rapport avec Marichette mais vise une nouvelle quête: former une nouvelle communauté. Cet ordre perturbé est l'indice que Marichette n'est pas l'objet de la quête puisque la Transfiguration du héros succède aux Noces. La "terre promise" est donc la quête réelle du héros qui fonde un village de colonisation. Dès lors, il devient de plus en plus malaisé d'établir une analogie quelconque entre le roman de Chauveau et les contes merveilleux russes; Charles Guérin est un roman canadien-français qui s'inscrit dans un certain contexte historique, social, politique, économique en fonction duquel il se donne à lire.

Cette analyse qui voulait «poser le problème de la priorité des formes sur celles des contenus" n'aboutit qu'à une grande confusion, les niveaux narratif et discursif n'étant pas distingués, pas plus que ne le sont les différentes séquences narratives, de sorte qu'action des personnages 
et discours du narrateur sont jugés équivalents tandis que les actions, détachées des exécutants, sont enchaînées les unes aux autres arbitrairement, d'où l'incohérence de la chaîne réalisée.

Le modèle de Propp, parce qu'il ne s'intéresse qu'au récit et le réduit à une chaîne unique de fonctions, offre des possibilités d'utilisation fort limitées mais nullement exploitées par l'analyse dont l'objectif ne vise que la répétition du schéma théorique. Une telle option ne peut déboucher sur d'autre conclusion que celle proposée, soit une affirmation systématique et péremptoire de la supériorité de ce mode d'approche du texte et une condamnation sans appel des méthodes "psycho-sociologiques" moins soucieuses des formes que du contenu et dont la caractéristique est une tendance à établir un lien entre roman et société.

Une analyse basée sur le modèle de Propp, mais bien conduite, aurait éventuellement pu tirer certaines conclusions intéressantes à partir de la conformité ou de la non-conformité du texte au modèle. Ainsi les fonctions manquantes 16 et 17 dans l'échantillon que nous avons présenté auraient pu être l'objet de commentaires. Que l'affrontement entre les protagonistes soit évincé du roman on pourrait y voir là un élément structural caractéristique et signifiant de la littérature canadienne-française, phénomène qui demanderait à être confirmé par une étude des textes complémentaires. Que, par ailleurs, les processus de possession (héritage) et dépossession (Clorinde au couvent) de Charles Guérin soient l'effet d'un hasard ou d'une force surnaturelle et non pas la manifestation du pouvoir du sujet, nous avons là des indices d'un système de valorisation des individus passifs opposés aux individus dynamiques; c'est donc là encore un lieu de signification qui aurait pu être exploité de même que l'inversion des fonctions Noces et Transfiguration qui fait de Marichette non pas la fin dans cette quête mais bien un simple moyen puisque la quête réelle est la colonisation.

CEuvre et auteur étant immergés dans un contexte historique, social, politique et économique donné, c'est en terme de production et de consommation qu'il faut envisager le texte. Le texte est produit et diffusé pour être lu, c'est un message et ceci est d'autant plus apparent dans le cas précis de Charles Guérin que pour en assurer une diffusion à plus grande échelle, un système est créé afin de le prendre en charge et c'est bien ce qu'affirme l'éditeur Cherrier assurant, en 1853, qu'il est le premier à acheter une œuvre littéraire. II ne s'agit pas là d'un fait anodin car à cette époque le thème du retour à la terre est largement diffusé par les journaux, les discours des élites laïques, les prônes et mandements de l'élite religieuse, et est fortement encouragé par l'élite anglaise quí, par le biais dés évêques canadiens-français, distribue fonds et publicité vantant les bienfaits de la vie à la campagne. Cette idéologie se retrouve jusque dans les manuels scolaires comme en témoigne cet extrait d'un manuel de pédagogie publié en $1865:$ : [...] détournons-les [les jeunes] de l'envie d'abandonner le séjour paisible et moral des champs pour les dangers et les séductions des villes; [...] enfin, prêchons-leur sur tous les tons l'importance vitale pour 
les Canadiens-français de s'emparer des terres vacantes et d'y fonder de nouvelles paroisses. Faisons-leur envisager cette colonisation de nos terres incultes comme un entreprise patriotique, comme une question de vie ou de mort pour notre race... ${ }^{7}$ ".

Charles Guerin appartient donc à un système culturel tout entier axé vers le retour à la terre des Canadiens français et c'est dans ce sens que Charles Guérin doit être lu. II n'y a aucune liberté d'interprétation puisque tous les constituants du texte sont orientés vers cette fin particulière. Dans cet univers particulier, les systèmes narratif et discursif manifestent l'échec et le succès du héros Charles Guérin en rapport avec des lieux précis : ville et campagne. Charles échoue en ville tandis que la campagne garantit la réussite individuelle et collective, réussite liée à la culture du sol et à la dispersion dans l'espace. L'échec du héros dans la gestion de l'entreprise industrielle de la Rivière aux Écrevisses suivi d'une dépossession (ses biens étant acquis par l'anglais Wagnaër), dépossession non suivie de lutte manifestant le désir de reconquérir ces biens perdus sont autant d'indices révélateurs de la fonction de ce roman qui, en orientant son héros vers la colonisation tandis que l'industrie est abandonnée à l'anglais, soutient, étoffe, renforce une idéologie particulière à une époque donnée.

Ainsi la recherche des «formes " qui se veut autre chose qu'un simple plaisir d'esthète ne peut se concevoir que subordonnée à la "lecture » de l'œuvre littéraire qui n'a ce statut, donc d'existence, que par sa fonction.

Janine Frot, Université de Sherbrooke.

1. Yvon Boucher, Fonctions et séquences dans "Charles Guérin" de Chauveau, Montréal, éd. Marc-Aimé Guérin, 1973.

2. P.J.O. Chauveau, Charles Guérin, Québec, éd. Marc-Aimé Guérin, 1973, page 171.

3. V. Propp. Morphologie du conte, Paris, Gallimard, 1970, p. 157, 158.

4. Propp, op. cit., page 36.

5. R. Barthes, Introduction à l'analyse structurale des récits, Paris, Seuil, “Communications », numéro 8, 1966, p. 17.

6. Propp, op. cit., p. 38.

7. Cité dans Fernand Dumont et collaborateurs, Idéologies au Canada français de 1850 à 1900, Québec, P.U.L., 1971, p. 36. 